\title{
Adaptive Tracking and Regulation of a Wheeled Mobile Robot With Controller/Update Law Modularity
}

\author{
W. E. Dixon, M. S. de Queiroz, D. M. Dawson, and T. J. Flynn
}

\begin{abstract}
A new adaptive controller is developed for wheeled mobile robots with parametric uncertainty in the dynamic model. The main theoretical contribution is the modular manner in which the control law and parameter update law are designed. This feature allows for design flexibility in the selection of the update law, and can be exploited to improve the transient response of the adaptive controller. The proposed controller also has the important feature of being applicable to both the tracking and regulation problems. The modularity of the adaptive controller is experimentally demonstrated on a K2A Cybermotion mobile robot that has been modified to allow for the implementation of torque-level control inputs. In particular, the adaptive controller with a gradient update law is evaluated vis-à-vis a least-squares update law.
\end{abstract}

Index Terms-Adaptive control, input-output stability, Lyapunov methods, least squares methods, mobile robot dynamics.

\section{INTRODUCTION}

$\mathbf{O}$ VER the last decade, the problem of regulating nonholonomic systems has been heavily targeted by control researchers due to the theoretically challenging nature of the problem. Specifically, due to the structure of the governing differential equations of the underactuated nonlinear system, the regulation problem cannot be solved via a smooth, time-invariant pure state feedback law due to the implications of Brockett's condition [2]. In addition to the regulation problem for the wheeled mobile robot (WMR), researchers have also targeted the more practical tracking control problem (which includes the path following problem as a subset). From a review of literature (see [3], [4], [8], [18], [20], [22]-[24] and the references therein), it can be observed that: i) most of the tracking controllers do not solve the regulation problem due to restrictions on the reference model trajectory signals; ii) most control designs rely heavily on the use of Barbalat's Lemma and its extensions during the kinematic stability analysis (i.e., the Lyapunov derivative is negative semidefinite

Manuscript received November 21, 2002. Manuscript received in final form May 27, 2003. Recommended by Associate Editor Y. Jin. This work was supported in part by the U.S. DOE Office of Biological and Environmental Research (OBER) Environmental Management Sciences Program (EMSP) Project ID 82797 at ORNL for the DOE Office of Science (SC), a subcontract to ORNL by the Florida Department of Citrus through the University of Florida, and by a U.S. NSF Grant DMI-9457967, ONR Grant N00014-99-1-0589, a DOC Grant, and an ARO Automotive Center Grant.

W. E. Dixon is with the Engineering Science and Tech. Div.-Robotics, Oak Ridge National Laboratory, Oak Ridge, TN 37831 USA (e-mail: dixonwe@ornl.gov).

M. S. de Queiroz is with the Department of Mechanical Engineering, Louisiana State University, Baton Rouge, LA 70803 USA.

D. M. Dawson is with the Department of Electrical and Computer Engineering, Clemson University, Clemson, SC 29634 USA.

T. J. Flynn is with the Imagery Technology \& Systems Division, Science Applications International Corporation, Tucson, AZ 85711 USA.

Digital Object Identifier 10.1109/TCST.2003.819587 in the system states as opposed to negative definite); iii) some of the kinematic controllers are not differentiable (e.g., see the kinematic controller developed in [18]), and hence, the standard integrator backstepping procedure cannot be used to incorporate the mechanical dynamics (see the discussion in [18]); iv) few results adaptively compensate for parametric uncertainty (e.g., payload mass, friction coefficients) in the dynamic model of the WMR; and v) all of the adaptive control results rely on standard gradient adaptive update laws.

To address some of the above issues, Dixon et al. [9] developed a differentiable kinematic control law that utilizes a dynamic oscillator-like control term to obtain a global uniformly ultimately bounded solution for the unified WMR tracking and regulation problems. Since the proposed kinematic controller is differentiable, standard backstepping techniques were used to design a nonlinear robust controller that rejects uncertainty associated with the dynamic model. In [10], Dixon et al. redesigned the dynamic oscillator of [9] to achieve global adaptive tracking and regulation control. In [11], Dong et al. exploited the differentiable kinematic control structure proposed in [23] to construct a global adaptive asymptotic tracking control law for a class of nonholonomic systems; however, the Lyapunov derivative for the controllers in [10] and [11] are negative semi-definite in the system states and position/velocity tracking error-based gradient adaptive update laws were required.

In contrast to the adaptive controllers for WMR's, several adaptive control results have been formulated for robot manipulators that explore new methods of parameter estimation. Most of this research has exploited Lyapunov-based techniques (i.e., the controller and the adaptive update law are designed in conjunction via a single Lyapunov function); however, the Lyapunov-based approach tends to restrict the design of the adaptive update law. For example, many of the previous adaptive controllers are restricted to utilizing position/velocity tracking error based gradient update laws. However, motivated by the fact that gradient update laws often exhibit slow parameter convergence (and hence, may retard the transient performance of the system) several researchers have explored control designs that incorporate other forms of update laws. Specifically, Slotine et al. [25] constructed a prediction error term as the difference between an estimated, filtered version of the robot dynamics and a filtered version of the input torque, and then developed a composite adaptive control law as the composite sum of a least-squares update law driven by the prediction error and a modified gradient update law driven by the link position/velocity tracking error. Although composite adaptive controllers have been experimentally proven (e.g., see [28]) to yield faster parameter convergence and improved transient response, the structure of the adaptive update law is still rather inflexible. In contrast to 
the Lyapunov-based approach given in [25], Leal et al. [14] utilized the flexibility ${ }^{1}$ provided by previous passivity-based adaptive control designs to construct a modified least-squares update law with the link position/velocity tracking error as the input. A modified least-squares update law based on the link position/velocity tracking error was also proposed by Sadegh et al. [21] in the design of an exponentially stable desired compensation adaptation law (DCAL) based controller, provided the desired regression matrix satisfies a semipersistency of excitation condition. In [26], Tang et al. developed an adaptive controller which included the standard gradient update law, the composite adaptation update law, and an averaging gradient update law as special cases.

In addition to the Lyapunov and passivity-based approaches given above, some research has exploited estimation-based approaches. Although these efforts have mainly targeted linear systems, estimation-based approaches allow for further flexibility in the construction of parameter update laws (e.g., prediction error-based gradient or least squares update laws can be designed) due to the modular design of the controller and the update law. For example, Middleton et al. [19] utilized a modular estimation-based approach to augment the adaptive computed torque controller of [5] with additional terms which allowed the closed-loop error system to be written as a stable, strictly proper, transfer function with the link position tracking error as the output and a prediction error related term as the input. The controller given in [19] enabled link position tracking and controller/update law modularity in the sense that any parameter update law could be used as long as its design ensured that: i) the parameter estimates remain bounded; ii) the prediction error is square integrable; and iii) the estimated inertia matrix is positive-definite (i.e., a projection-type algorithm is required in the parameter update law). In [13], Krstic et al. utilized nonlinear damping [12] to extend previous linear estimation-based techniques to a class of parametric-strict-feedback nonlinear systems; however, this class of systems, does not encompass the robot dynamics due to coupling terms in the inertia matrix. However, motivated by the development given in [13], de Queiroz et al. [6] developed an adaptive link position tracking controller for robot manipulators which achieves controller/update law modularity. Although the result was similar to the result given in [19], the controller developed in [6] does not require the estimated inertia matrix to be positive-definite and does not require the online calculation of the inverse of the estimated inertia matrix.

An adaptive torque control input for WMRs is developed in this paper to solve the unified tracking and regulation control problem. This result is achieved despite the presence of parametric uncertainty in the system dynamics. To compensate for parametric uncertainty in the system, a nonlinear damping based strategy, inspired by [6], is employed to provide input-to-state stability (ISS) of the controller. The ISS result yields a modular adaptive controller that allows the adaptive update law to be designed in a modular manner with respect to the feedback

\footnotetext{
${ }^{1}$ The passivity-based adaptive controllers provide some flexibility in the design of the update law; however, the update law must be designed to satisfy a passive mapping condition.
}

controller; hence, allowing for improved design flexibility. That is, provided a prediction error-based update law ensures the parameter estimate vector is bounded, all of the signals are proven to be bounded through an ISS analysis. An additional stability analysis is provided to prove that if the adaptive update law is designed such that the prediction error is square-integrable and the estimated inertia matrix is positive-definite, then the WMR tracking and regulation errors are globally asymptotically forced to a constant that can be made arbitrarily small. To facilitate this result, we exploit the kinematic control structure of [9]. The structure of the kinematic controller is crucial to the development of the modular adaptive controller. Specifically, this approach is motivated by the fact that the kinematic controller is differentiable; thus, enabling integrator backstepping to incorporate the effects of the system dynamics. The approach ensures the transformed states of the system are negative-definite in the time derivative of a radially unbounded nonnegative function. This concept is a key advantage over many of the current WMR designs, which are negative semidefinite in the system states and require tools such as extended Barbalat's Lemma to prove stability, because it can be fused with nonlinear damping techniques to facilitate an ISS result. To highlight the modular nature of the adaptive controller, experimental results are provided to demonstrate that the controller can be implemented with a prediction error based gradient update law and then implemented with the same control gains with a prediction error based least-squares update law to achieve similar performance.

\section{KinEMATIC MODEL}

The kinematic model for the so-called kinematic wheel under the nonholonomic constraint of pure rolling and nonslipping is given as follows:

$$
\dot{q}=S(q) v
$$

where $q(t), \dot{q}(t) \in R^{3}$ are defined as

$$
q=\left[\begin{array}{lll}
x_{c} & y_{c} & \theta
\end{array}\right]^{T} \quad \dot{q}=\left[\begin{array}{lll}
\dot{x}_{c} & \dot{y}_{c} & \dot{\theta}
\end{array}\right]^{T}
$$

$x_{c}(t), y_{c}(t)$, and $\theta(t) \in R$ denote the position and orientation, respectively, of the center of mass (COM) of the WMR, $\dot{x}_{c}(t), \dot{y}_{c}(t)$ denote the Cartesian components of the linear velocity of the COM, and $\dot{\theta}(t) \in R$ denotes the angular velocity of the COM. In (1), the matrix $S(q) \in R^{3 \times 2}$ is defined as follows:

$$
S(q)=\left[\begin{array}{cc}
\cos \theta & 0 \\
\sin \theta & 0 \\
0 & 1
\end{array}\right]
$$

and the velocity vector $v(t) \in R^{2}$ is defined as

$$
v=\left[\begin{array}{ll}
v_{1} & v_{2}
\end{array}\right]^{T}=\left[\begin{array}{ll}
v_{l} & \dot{\theta}
\end{array}\right]^{T}
$$

with $v_{l}(t) \in R$ denoting the linear velocity of the COM of the WMR.

\section{OPEN-LOOP ERROR SYSTEM}

To formulate the tracking control problem, we define the following time-varying reference model

$$
\dot{q}_{r}=S\left(q_{r}\right) v_{r}
$$


where $S(\cdot)$ was defined in (3), $q_{r}(t)=\left[x_{r c}(t), y_{r c}(t)\right.$, $\left.\theta_{r}(t)\right]^{T} \in R^{3}$ denotes the desired time-varying position and orientation trajectory, and $v_{r}(t)=\left[v_{r 1}(t) v_{r 2}(t)\right]^{T} \in R^{2}$ denotes the reference time-varying linear and angular velocity. With regard to (5), it is assumed that the signal $v_{r}(t)$ is constructed to produce the desired motion and that $v_{r}(t), \dot{v}_{r}(t), q_{r}(t)$, and $\dot{q}_{r}(t)$ are bounded for all time. See Remark 1.3 of [8] for an example of one method for selecting $v_{r}(t)$ such that a desired Cartesian path is generated.

To facilitate the subsequent control synthesis and the corresponding stability proof, the following global invertible transformation is defined [8]

$$
\left[\begin{array}{c}
w \\
z_{1} \\
z_{2}
\end{array}\right]=\left[\begin{array}{ccc}
-\tilde{\theta} \cos \theta+2 \sin \theta & -\tilde{\theta} \sin \theta-2 \cos \theta & 0 \\
0 & 0 & 1 \\
\cos \theta & \sin \theta & 0
\end{array}\right]\left[\begin{array}{c}
\tilde{x} \\
\tilde{y} \\
\tilde{\theta}
\end{array}\right]
$$

where $w(t) \in R$ and $z(t)=\left[z_{1}(t) z_{2}(t)\right]^{T} \in R^{2}$ are auxiliary tracking error variables, and $\tilde{x}(t), \tilde{y}(t), \tilde{\theta}(t) \in R$ denote the difference between the actual Cartesian position and orientation of the $\mathrm{COM}$ and the reference position and orientation of the COM as follows:

$$
\tilde{x}=x_{c}-x_{r c} \quad \tilde{y}=y_{c}-y_{r c} \quad \tilde{\theta}=\theta-\theta_{r} .
$$

After taking the time derivative of (6) and using (1)-(7), the tracking error dynamics can be expressed in terms of the auxiliary variables defined in (6) as follows [8]:

$$
\begin{aligned}
\dot{w} & =u^{T} J^{T} z+f \\
\dot{z} & =u
\end{aligned}
$$

where $J \in R^{2 \times 2}$ denotes a skew-symmetric matrix defined as

$$
J=\left[\begin{array}{cc}
0 & -1 \\
1 & 0
\end{array}\right]
$$

and $f\left(z, v_{r}\right) \in R$ is defined as

$$
f=2\left(v_{r 2} z_{2}-v_{r 1} \sin z_{1}\right) .
$$

The auxiliary variable $u(t)=\left[u_{1}(t) u_{2}(t)\right]^{T} \in R^{2}$ introduced in (8) is defined in terms of the WMR position and orientation, linear and angular velocities, and the reference trajectory as follows:

$$
u=T^{-1} v-\left[\begin{array}{c}
v_{r 2} \\
v_{r 1} \cos \tilde{\theta}
\end{array}\right] \quad v=T u+\Pi .
$$

In (11), the global invertible matrix $T(q) \in R^{2 \times 2}$ is defined as follows:

$$
T=\left[\begin{array}{cc}
(\tilde{x} \sin \theta-\tilde{y} \cos \theta) & 1 \\
1 & 0
\end{array}\right]
$$

and $\Pi\left(q, q_{r}, v_{r}\right) \in R^{2}$ is defined as

$$
\Pi=\left[\begin{array}{l}
v_{r 1} \cos \tilde{\theta}+v_{r 2}(\tilde{x} \sin \theta-\tilde{y} \cos \theta) \\
v_{r 2}
\end{array}\right] .
$$

\section{DYNAMIC MODEL}

The WMR dynamic model can be expressed in the following form [8]

$$
M^{*} \dot{u}+V_{m}^{*} u+N^{*}=B^{*} \tau
$$

where

$$
\begin{aligned}
M^{*}=T^{T} M T, & B^{*}=T^{T} B, \\
V_{m}^{*} & =T^{T} M \dot{T}, \quad N^{*}=T^{T}(F+M \dot{\Pi}) .
\end{aligned}
$$

In (15), $M \in R^{2 \times 2}$ represents the constant, positive-definite inertia matrix, $F\left(u, q, q_{r}, v_{r}\right) \in R^{2}$ represents the friction effects, $\tau(t) \in R^{2}$ represents the torque input vector, $B \in R^{2 \times 2}$ represents a known, constant global invertible input matrix that governs torque transmission to the wheels (see [8] for explicit examples of $B)$, and $T(q)$ and $\Pi\left(q, q_{r}, v_{r}\right)$ are defined in (12) and (13), respectively. The dynamic equation of (14) exhibits the following properties which will be employed during the subsequent control development and stability analysis.

Property 1: The transformed inertia matrix $M^{*}(q)$ is symmetric, positive-definite, and satisfies the following inequalities [8]

$$
m_{1}\|\xi\|^{2} \leq \xi^{T} M^{*} \xi \leq m_{2}(z, w)\|\xi\|^{2} \quad \forall \xi \in R^{2}
$$

where $m_{1} \in R$ is a known positive constant, $m_{2}(z, w) \in R$ is a known, positive bounding function which is assumed to be bounded provided $z(t)$ and $w(t)$ are bounded $\left(\dot{m}_{2}(z, w, \dot{z}, \dot{w})\right.$ is also assumed to be bounded provided $z(t), \dot{z}(t), w(t)$, and $\dot{w}(t)$ are bounded), and $\|\cdot\|$ is the standard Euclidean norm.

Property 2: A skew-symmetric relationship exists between the transformed inertia matrix and $V_{m}^{*}(q, \dot{q})$ as follows:

$$
\xi^{T}\left(\frac{1}{2} \dot{M}^{*}-V_{m}^{*}\right) \xi=0 \quad \forall \xi \in R^{2}
$$

where $\dot{M}^{*}(q)$ represents the time derivative of the transformed inertia matrix.

Property 3: The robot dynamics given in (14) can be linearly parameterized as follows:

$$
Y \Phi=M^{*} \dot{u}+V_{m}^{*} u+N^{*}
$$

where $\Phi \in R^{p}$ contains the unknown constant mechanical parameters (i.e., inertia, mass, and friction effects) and $Y(u, \dot{u}) \in R^{2 \times p}$ denotes the known regression matrix. The following linear parameterization can be formulated

$$
Y_{s} \Phi=M^{*} \dot{u}_{d}+V_{m}^{*} u_{d}+N^{*}
$$

where $Y_{s}\left(u, u_{d}, \dot{u}_{d}\right) \in R^{2 \times p}$ denotes a known regression matrix, $\Phi$ is the same unknown constant parameter vector given in (18), and $\dot{u}_{d}(t) \in R^{2}$ represents the time derivative of the subsequently designed input $u_{d}(t) \in R^{2}$.

\section{Control Development}

\section{A. Control Objective}

The objective in this paper is to design an adaptive controller that solves the unified tracking and regulation problems for a WMR under the additional constraint of parametric uncertainty for the robot dynamics of (14) such that a modularity is achieved in the design of the controller and the parameter update law. To quantify the error between the parameter estimate generated by the modular adaptive update law and the actual parameters of 
the WMR dynamic model, a parameter estimation error vector is defined as follows:

$$
\tilde{\Phi}=\Phi-\hat{\Phi}
$$

where $\hat{\Phi}(t) \in R^{p}$ is a dynamic estimate of $\Phi$, defined in (18). Motivated by the desire to facilitate a modular adaptive control scheme that is independent of acceleration measurements, the measurable prediction error signal $\varepsilon(t) \in R^{2}$ is defined as follows:

$$
\varepsilon=Y_{f} \tilde{\Phi}=Y_{f} \Phi-Y_{f} \hat{\Phi}=\tau_{f}-Y_{f} \hat{\Phi} .
$$

The filtered regression matrix, denoted by $Y_{f}(u) \in R^{2 \times p}$, introduced in (21) is defined by the following differential equation and initial condition

$$
\dot{Y}_{f}+\beta Y_{f}=\beta Y, \quad Y_{f}(u(0))=0
$$

where $Y(u, \dot{u})$ was defined in (18) (see [16] for details regarding an acceleration independent formulation of $\left.Y_{f}(u)\right), \beta \in R$ is a positive control term, and the filtered torque $\tau_{f}(t) \in R^{2}$ is generated by the following differential equation and initial condition

$$
\dot{\tau}_{f}+\beta \tau_{f}=\beta B^{*} \tau, \quad \tau_{f}(0)=0
$$

where $\tau(t)$ and $B^{*}(q)$ are defined in (14) and (15), respectively.

To achieve the objective of simultaneously solving the tracking and regulation problems, a control strategy is employed that exploits a dynamic oscillator-like structure. To facilitate this control design, an auxiliary error signal, denoted by $\tilde{z}(t) \in R^{2}$, is defined as the difference between the subsequently designed dynamic oscillator-like signal $z_{d}(t) \in R^{2}$ and the transformed variable $z(t)$, defined in (6), as follows:

$$
\tilde{z}=z_{d}-z \text {. }
$$

A backstepping error signal, denoted by $\eta(t) \in R^{2}$, is defined to quantify the mismatch between the kinematic velocity signal $u(t)$ and the subsequently designed desired kinematic velocity input, denoted by $u_{d}(t)$, as follows:

$$
\eta=u_{d}-u \text {. }
$$

\section{B. Control Design}

Based on the open-loop kinematic system given in (8) and the subsequent stability analysis, we design $u_{d}(t)$ [9]

$$
u_{d}=u_{a}-k_{2} m_{2} z
$$

where $m_{2}(z, w)$ was given in (16), and $k_{2} \in R$ is a positive, constant control gain. The auxiliary control term $u_{a}(t) \in R^{2}$ introduced in (26) is defined as

$$
u_{a}=\left(\frac{k_{1} m_{2} w+f}{\delta_{d}^{2}}\right) J z_{d}+\Omega_{1} z_{d}
$$

where the auxiliary signal $z_{d}(t)$ is defined by the following dynamic oscillator-like relationship

$$
\begin{aligned}
\dot{z}_{d} & =\frac{\dot{\delta}_{d}}{\delta_{d}} z_{d}+\left(\frac{k_{1} m_{2} w+f}{\delta_{d}^{2}}+w \Omega_{1}\right) J z_{d} \\
z_{d}^{T}(0) z_{d}(0) & =\delta_{d}^{2}(0)
\end{aligned}
$$

the auxiliary terms $\Omega_{1}\left(w, z, v_{r}\right) \in R$ and $\delta_{d}(t) \in R$ are defined as

$$
\Omega_{1}=k_{2} m_{2}+\frac{\dot{\delta}_{d}}{\delta_{d}}+w\left(\frac{k_{1} m_{2} w+f}{\delta_{d}^{2}}\right)
$$

and

$$
\delta_{d}=\alpha_{0} \exp \left(-\alpha_{1} t\right)+\varepsilon_{1}
$$

respectively, $k_{1}, \alpha_{0}, \alpha_{1}, \varepsilon_{1} \in R$ are positive, constant control gains, and $f\left(z, v_{r}\right)$ was defined in (10). Motivation for the structure of (28) is obtained by taking the time derivative of $z_{d}^{T}(t) z_{d}(t)$ as follows

$$
\frac{d}{d t}\left(z_{d}^{T} z_{d}\right)=2 z_{d}^{T}\left(\frac{\dot{\delta}_{d}}{\delta_{d}} z_{d}+\left(\frac{k_{1} m_{2} w+f}{\delta_{d}^{2}}+w \Omega_{1}\right) J z_{d}\right)
$$

where (28) has been utilized. After noting that the matrix $J$ of (9) is skew symmetric, we can rewrite (31) as

$$
\frac{d}{d t}\left(z_{d}^{T} z_{d}\right)=2 \frac{\dot{\delta}_{d}}{\delta_{d}} z_{d}^{T} z_{d} .
$$

As result of the selection of the initial conditions given in (28), it is easy to verify that

$$
z_{d}^{T} z_{d}=\left\|z_{d}\right\|^{2}=\delta_{d}^{2}
$$

is a unique solution to the differential equation given in (32) (see [9] for motivation regarding the design of $\delta_{d}(t)$ ).

Based on the transformed dynamic model given by (14) and the subsequent stability analysis, we design the control torque input $\tau(t)$ as follows:

$$
\tau=\left(B^{*}\right)^{-1}\left(\tau^{*}+Y_{s} \hat{\Phi}+k_{a} m_{2} \eta\right)
$$

where $\tau^{*}(t) \in R^{2}$ is defined as

$$
\begin{aligned}
\tau^{*}= & {\left[\frac{1}{\beta} Y_{f} \dot{\hat{\Phi}}+\left(\dot{\hat{M}}^{*}-\hat{V}_{m}^{*}\right) \eta\right]+k_{n 1}\left\|Y_{s}\right\|_{i \infty}^{2} \eta } \\
& +k_{n 2}\left\|\frac{1}{\beta} Y_{f} \dot{\hat{\Phi}}\right\|^{2} \eta+k_{n 3}\left\|\left(\dot{\hat{M}}^{*}-\hat{V}_{m}^{*}\right) \eta\right\|^{2} \eta \\
& +k_{n 4}\|J z w\|^{2} \eta+k_{n 5}\|\tilde{z}\|^{2} \eta
\end{aligned}
$$

where $m_{2}(z, w), Y_{s}\left(u, u_{d}, \dot{u}_{d}\right), \hat{\Phi}(t)$, and $Y_{f}(u)$ are defined in (16), (19), (20), and (22), respectively, $\dot{\hat{\Phi}}(t) \in R^{p}$ (which includes $\hat{\bar{M}}^{*}(\cdot)$ and $\left.\hat{V}_{m}^{*}(\cdot)\right)$ denotes the time derivative of the dynamic estimate $\hat{\Phi}(t)$, and $k_{a}, k_{n i} \in R \forall i=1,2, \ldots, 5$ are positive constant control gains. The bracketed control terms given in (35) are incorporated in the control design to cancel similar terms in the subsequent stability analysis. The remaining terms of (35) are incorporated to facilitate the input-to-state stability property of the closed-loop system with respect to $\tilde{\Phi}(t)$.

\section{Closed-Loop Error Systems}

To facilitate the closed-loop error system development, the auxiliary control input signal $u_{d}(t)$ is injected into the open-loop dynamics of $w(t)$ given by (8) by adding and subtracting the term $u_{d}^{T}(t) J z(t)$ to the right-hand side (RHS) of (8) to obtain the following expression

$$
\dot{w}=-u_{d}^{T} J z+\eta^{T} J z+f
$$


where (25) was utilized. After substituting (26) for $u_{d}(t)$, adding and subtracting $u_{a}^{T}(t) J z_{d}(t)$ to the resulting expression, utilizing (24), and exploiting the skew symmetry of $J$ defined in (9), the dynamics for $w(t)$ can be determined as

$$
\dot{w}=-u_{a}^{T} J z_{d}+u_{a}^{T} J \tilde{z}+\eta^{T} J z+f .
$$

Finally, by substituting (27) for only the first occurrence of $u_{a}(t)$ in (37) and then utilizing the equality given by (33), the skew symmetry of $J$ defined in (9), and the fact that $J^{T} J=I_{2}$ (Note that $I_{2}$ denotes the standard $2 \times 2$ identity matrix), the following expression for the closed-loop error system for $w(t)$ can be obtained

$$
\dot{w}=-k_{1} m_{2} w+u_{a}^{T} J \tilde{z}+\eta^{T} J z .
$$

To determine the closed-loop error system for $\tilde{z}(t)$, we take the time derivative of (24), substitute (28) for $\dot{z}_{d}(t)$, and then substitute (8) for $\dot{z}(t)$ to obtain

$$
\dot{\tilde{z}}=\frac{\dot{\delta}_{d}}{\delta_{d}} z_{d}+\left(\frac{k_{1} m_{2} w+f}{\delta_{d}^{2}}+w \Omega_{1}\right) J z_{d}+\eta-u_{d}
$$

where the auxiliary control input $u_{d}(t)$ was injected by adding and subtracting $u_{d}(t)$ to the RHS of (39), and (25) was utilized. After substituting (26) for $u_{d}(t)$ and then substituting (27) for $u_{a}(t)$ in the resulting expression, we can rewrite (39) as follows:

$$
\dot{\tilde{z}}=\frac{\dot{\delta}_{d}}{\delta_{d}} z_{d}+w \Omega_{1} J z_{d}-\Omega_{1} z_{d}+k_{2} m_{2} z+\eta .
$$

After substituting (29) for only the second occurrence of $\Omega_{1}(t)$ in (40) and using the fact that $J J=-I_{2}$, the following simplified expression can be obtained

$$
\dot{\tilde{z}}=-k_{2} m_{2} \tilde{z}+w J\left[\left(\frac{k_{1} m_{2} w+f}{\delta_{d}^{2}}\right) J z_{d}+\Omega_{1} z_{d}\right]+\eta
$$

where (24) has been utilized. Since the bracketed term in (41) is equal to $u_{a}(t)$ defined in (27), the final expression for the closed-loop error system for $\dot{\tilde{z}}(t)$ can be expressed as

$$
\dot{\tilde{z}}=-k_{2} m_{2} \tilde{z}+w J u_{a}+\eta \text {. }
$$

To develop the closed-loop error system for $\eta(t)$, we take the time derivative of (25), substitute (14) for $\dot{u}(t)$, add and subtract $V_{m}^{*}(q, \dot{q}) u_{d}(t)$, and then rearrange the resulting expression as

$$
M^{*} \dot{\eta}=Y_{s} \Phi-V_{m}^{*} \eta-B^{*} \tau
$$

where (19) and (25) were utilized. After substituting (34) and (35) into (43), the following expression is obtained

$$
\begin{aligned}
M^{*} \dot{\eta}= & -V_{m}^{*} \eta+Y_{s} \tilde{\Phi}-k_{a} m_{2} \eta-\left(\dot{\hat{M}}^{*}-\hat{V}_{m}^{*}\right) \\
& -\frac{1}{\beta} Y_{f} \dot{\hat{\Phi}}-k_{n 2}\left\|\frac{1}{\beta} Y_{f} \dot{\hat{\Phi}}\right\|^{2}-k_{n 3}\left\|\left(\dot{\hat{M}}^{*}-\hat{V}_{m}^{*}\right) \eta\right\|^{2} \\
& -\left(k_{n 1}\left\|Y_{s}\right\|_{i \infty}^{2}+k_{n 4}\|J z w\|^{2}+k_{n 5}\|\tilde{z}\|^{2}\right) \eta
\end{aligned}
$$

where (20) was utilized.

\section{STABILITy ANALYSIS}

Based on the previous development, several results can now be proven. To facilitate a modular adaptive result, the first objective is to demonstrate that the controller yields ISS as indicated by the following theorem.

Theorem 1: Given the closed-loop error systems in (38), (42), and (44), if $\tilde{\Phi}(t) \in L_{\infty}\left[0, t_{f}\right)$ then all signals are bounded under closed-loop operation for $\left[0, t_{f}\right)$ where $t_{f}$ denotes the final time.

Based on the ISS result of Theorem 1 (see the Appendix for a proof), the modular adaptive unified tracking and regulation problem can be examined. Specifically, the position and orientation tracking errors can be proven to asymptotically approach an arbitrarily small positive constant, provided some adaptive update law, denoted by $\dot{\hat{\Phi}}(u, \varepsilon)$, is designed according to the following theorem.

Theorem 2: Any update law that ensures $\hat{\Phi}(t) \in$ $L_{\infty}\left[0, t_{f}\right), \hat{M}^{*}(q)$ is positive-definite, and $\varepsilon(t) \in L_{2}\left[0, t_{f}\right)$, can be used in conjunction with the controller developed in (34) and (35) to ensure that all signals are bounded during closed-loop operation for $t \in[0, \infty)$, and the position and orientation tracking errors $|\tilde{x}(t)|,|\tilde{y}(t)|,|\tilde{\theta}(t)|$ asymptotically approach a positive constant as follows:

$$
\lim _{t \rightarrow \infty}|\tilde{x}(t)|,|\tilde{y}(t)|,|\tilde{\theta}(t)|=\rho \varepsilon_{1}
$$

where $\rho \in R$ is a positive bounding constant, and $\varepsilon_{1}$ can be made arbitrarily small.

The proof for Theorem 2 (see the Appendix) requires that $\dot{\hat{\Phi}}(u, \varepsilon)$ be designed so that $\hat{\Phi}(t) \in L_{\infty}, \hat{M}^{*}(q)$ is positive-definite, and $\varepsilon(t) \in L_{2}$. A typical parameter adaptation algorithm which ensures that $\hat{\Phi}(t) \in L_{\infty}$ and $\varepsilon(t) \in L_{2}$ is given by the following gradient update law

$$
\dot{\hat{\Phi}}=\Gamma Y_{f}^{T} \varepsilon
$$

where $\Gamma \in R^{p \times p}$ is a positive-definite gain matrix. Another typical parameter adaptation algorithm satisfying the previous conditions is the following least-squares estimator

$$
\dot{\hat{\Phi}}=P Y_{f}^{T} \varepsilon, \quad \dot{P}=-P Y_{f}^{T} Y_{f} P
$$

where $P(t) \in R^{p \times p}$ is a time-varying symmetric matrix. To ensure that $\hat{M}^{*}(q)$ is positive-definite, a standard projection algorithm can be incorporated in the design of (46) and (47) (see [1] and [15]).

Remark 3: Based on the fact that no restrictions were placed on the reference trajectory $v_{r}(t)$ with the exception that $v_{r}(t), \dot{v}_{r}(t) \in L_{\infty}$, it is straightforward to prove that the tracking control result given in Theorem 2 is also valid for the regulation problem (i.e., $v_{r}(t)=0$ ).

\section{EXPERIMENTAL VERIFICATION}

\section{A. Experimental Configuration}

The developed modular adaptive tracking controller introduced in (34) and (35) was implemented on the modified K2A 


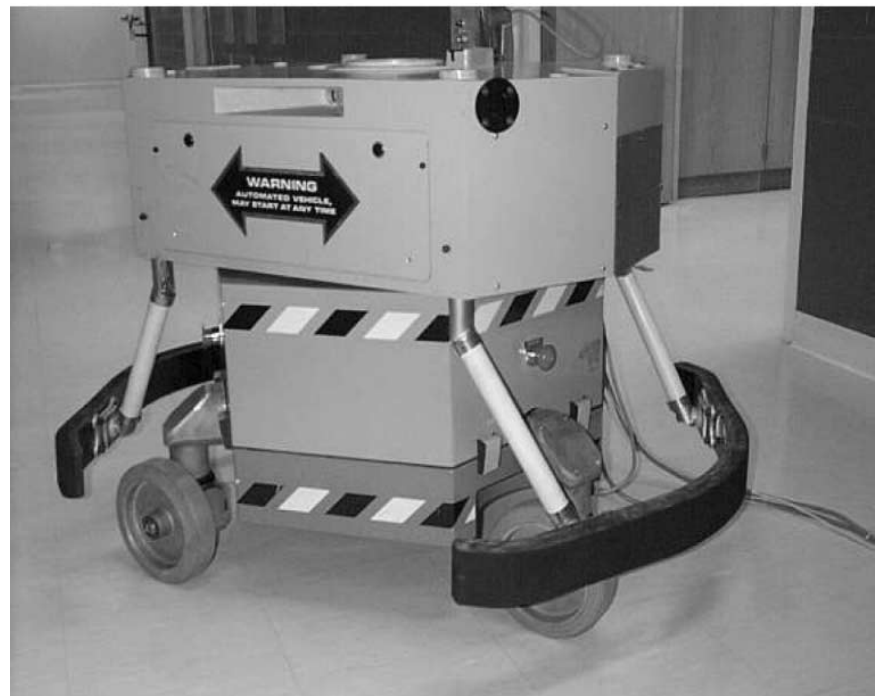

Fig. 1. K2A mobile robot testbed.

WMR manufactured by Cybermotion Inc. shown in Fig. 1. Permanent magnet dc motors provide steering and drive actuation through a 106:1 and a 96:1 gear coupling, respectively. The positions of the steering and drive motors are measured via Hewlett Packard (HEDS-9000) encoders with a resolution of $0.35 \mathrm{deg} /$ line, and velocity measurements were calculated via a filtered backward difference algorithm. A Pentium $133 \mathrm{MHz}$ PC operating under QNX (a real-time microkernel based operating system) hosts the control algorithm that was written in " $\mathrm{C}++$ ", and implemented using Qmotor 3.0 [17]. Data acquisition and control implementation were performed at a frequency of 1.0 $\mathrm{kHz}$ using the MultiQ I/O board. To measure the tracking error given in (7), the $x(t)$ and $y(t)$ coordinate and the orientation of the WMR are required. To obtain the position and orientation signals, the positions of the steer and drive motors were obtained from the encoder measurements. Based on the steer and drive motor positions, linear and angular velocity measurements were calculated via a filtered backward difference algorithm. Using the angular position measurement and the linear and angular velocity measurements, the relationship given in (1) was used to determine $\dot{x}(t)$ and $\dot{y}(t)$. A numerical integration routine was then applied to (1) to obtain the actual values of the Cartesian coordinates of the WMR. For simplicity the electrical dynamics of the system were ignored. That is, the computed torque is assumed to be statically related to the voltage input of the permanent magnet dc motors by a constant. For further details regarding the experimental testbed see [8].

The dynamics for the modified K2A WMR are given

$$
\frac{1}{r_{o}}\left[\begin{array}{cc}
1 & 0 \\
0 & \frac{L_{o}}{2}
\end{array}\right]\left[\begin{array}{l}
\tau_{1} \\
\tau_{2}
\end{array}\right]=\left[\begin{array}{c}
m_{o} \dot{v}_{1}+F_{d 1} v_{1} \\
I_{o} \dot{v}_{2}+F_{d 2} v_{2}
\end{array}\right]
$$

where $m_{o}=165 \mathrm{~kg}$ denotes the mass of the robot, $I_{o}=4.643$ $\mathrm{kg} \cdot \mathrm{m}^{2}$ denotes the inertia of the robot, $r_{o}=0.010 \mathrm{~m}$ denotes the radius of the wheels, $L_{o}=0.667 \mathrm{~m}$ denotes the length of the axis between the wheels, and the dynamic friction elements
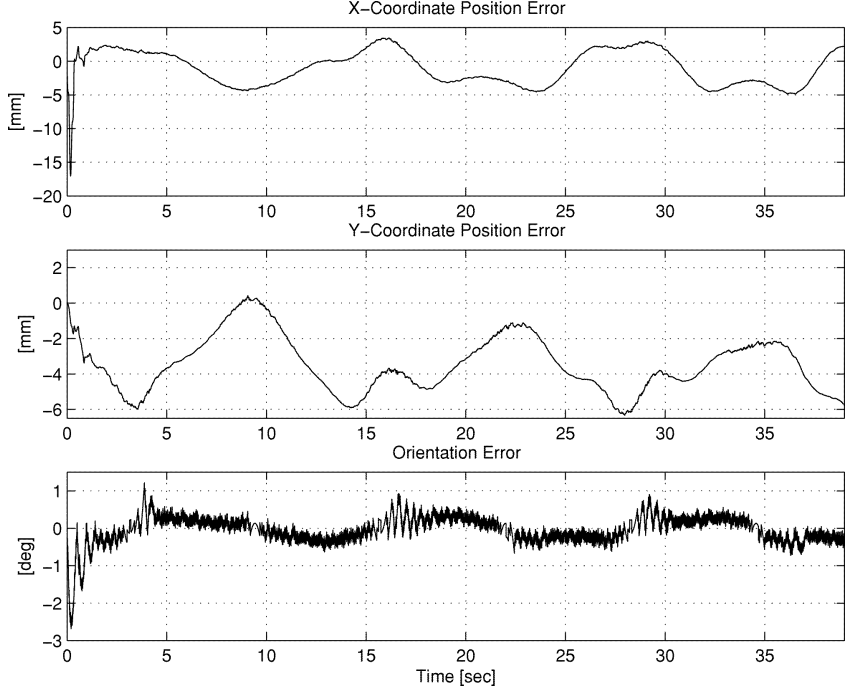

Fig. 2. Position and orientation tracking errors.

are denoted by $F_{d 1}$, and $F_{d 2}$. The desired reference linear and angular velocity were selected as

$$
\begin{aligned}
& v_{r 1}=0.2(1-\exp (-t))(\mathrm{m} / \mathrm{s}) \\
& v_{r 2}=0.4 \sin (0.5 t)(\mathrm{rad} / \mathrm{s})
\end{aligned}
$$

respectively, (the resulting reference time-varying Cartesian position and orientation is a sinusoidal trajectory with a peak-to-peak amplitude of approximately $0.6 \mathrm{~m}$ ).

\section{B. Experimental Results}

The controller introduced in (34) and (35) was first implemented with the prediction error based gradient update law given in (46). The control and adaptation gains were then tuned to achieve the best results in terms of steady-state tracking error. The control gains that resulted in the best performance are given

$$
\begin{aligned}
k_{1} & =0.665, \quad k_{2}=0.82, \quad k_{a}=\left[\begin{array}{cc}
0.65 & 0 \\
0 & 19.0
\end{array}\right] \\
k_{n 1} & =k_{n 2}=0.001, \quad k_{n 3}=k_{n 4}=0.1, \quad k_{n 5}=0.01 \\
\alpha_{0} & =0.014, \quad \alpha_{1}=27.5, \quad \varepsilon_{1}=1.0, \quad \varepsilon_{2}=0.75 .
\end{aligned}
$$

where the adaptation gains were selected as follows:

$$
\Gamma_{1}=0.00075, \quad \Gamma_{2}=\Gamma_{4}=0.0001, \quad \Gamma_{3}=0.00001 \text {. }
$$

Note that although $k_{a}$ of (34) was defined as a scalar constant, the values given in (50) and (51) were used to facilitate the "tuning" process. The position/orientation tracking error of the COM of the WMR and the associated control torque inputs are shown in Figs. 2 and 3, respectively. The control torque inputs plotted in Fig. 3 represent the torques applied by the motor multiplied by the gear ratio to represent the torque applied to the wheels. The parameter estimates are illustrated in Fig. 4. Based on the data for Fig. 2, the steady-state position/orientation tracking error can bounded as follows:

$$
|\tilde{x}|<5 \mathrm{~mm} \quad|\tilde{y}|<6.25 \mathrm{~mm} \quad|\tilde{\theta}|<1.0 \mathrm{deg} .
$$



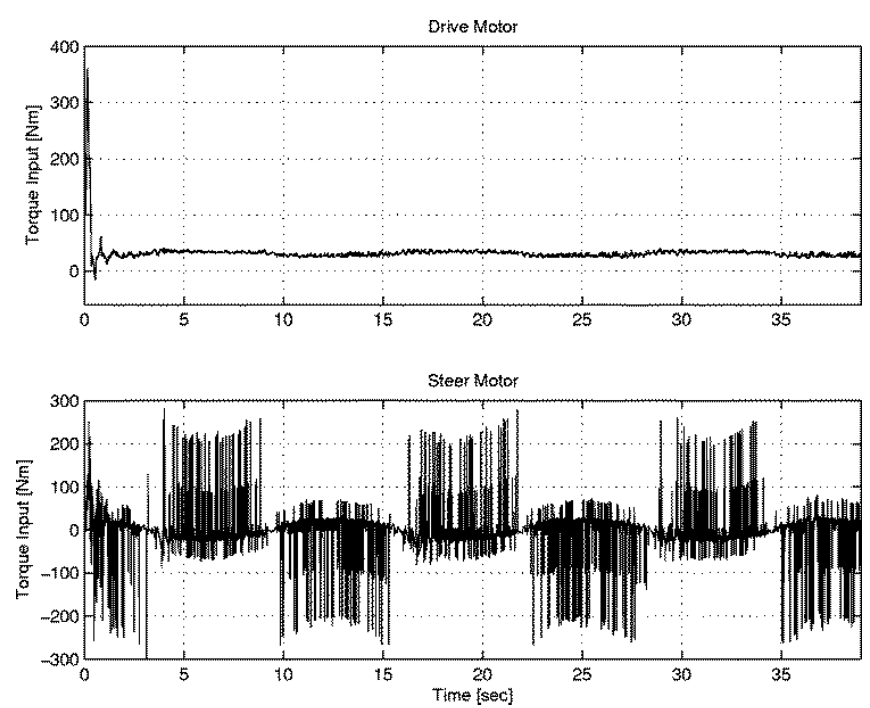

Fig. 3. Control torque input applied by the steer and drive motors.

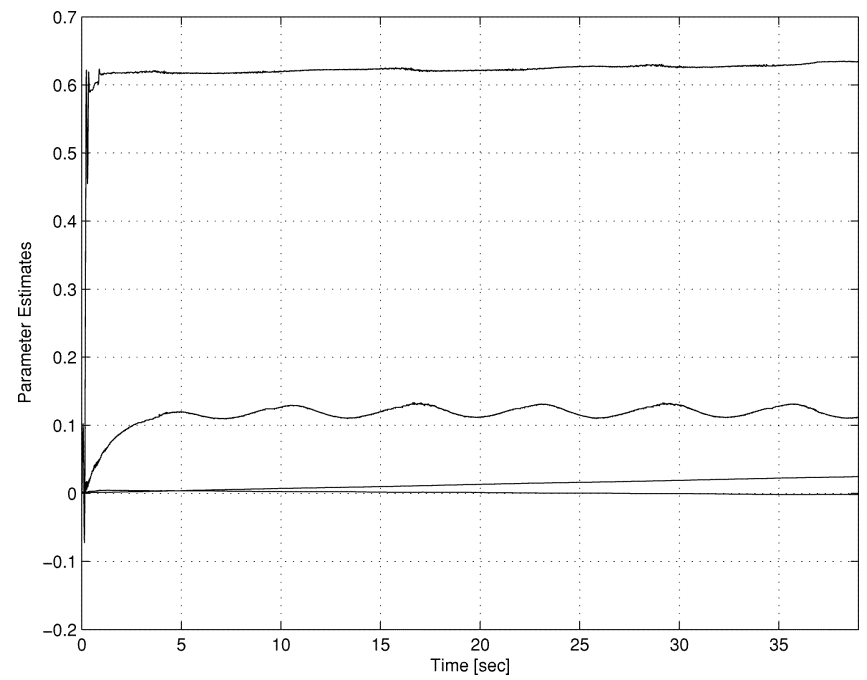

Fig. 4. Parameter estimates for the gradient update law.

To demonstrate modularity between the controller and the parameter update law, the controller was then implemented with the prediction error based least-squares update law given in (47). Without retuning the controller (i.e., using the same control gains as in (50)) the gradient update law was replaced with the least-squares estimator in (47) with the initial conditions for the symmetric matrix $P(t)$ selected as follows:

$$
P(0)=\left[\begin{array}{cccc}
0.3 & 10^{-5} & 0.025 & 0.01 \\
10^{-5} & 1.0 & 0.075 & 0.1 \\
0.025 & 0.075 & 1.75 & 0.025 \\
0.01 & 0.1 & 0.025 & 4
\end{array}\right] .
$$

The position/orientation tracking error of the COM of the WMR and the associated control torque inputs are shown in Figs. 5 and 6, respectively. The parameter estimates are illustrated in Fig. 7. Based on the data for Fig. 5, the steady-state position/orientation tracking error can be bounded as

$$
|\tilde{x}|<2.5 \mathrm{~mm} \quad|\tilde{y}|<5.0 \mathrm{~mm} \quad|\tilde{\theta}|<1.0 \mathrm{deg} .
$$
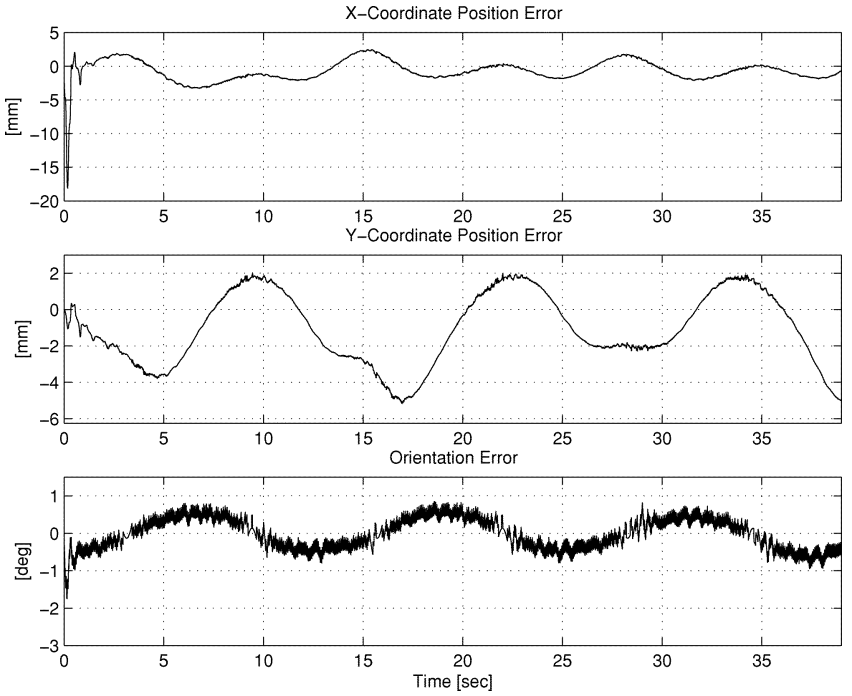

Fig. 5. Position and orientation tracking errors.
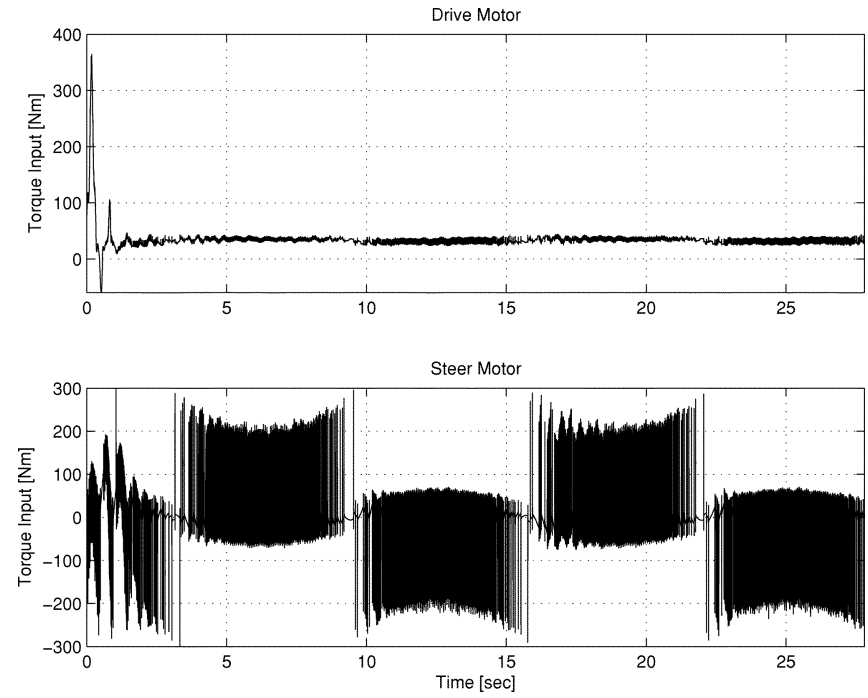

Fig. 6. Control torque input applied by the steer and drive motors.

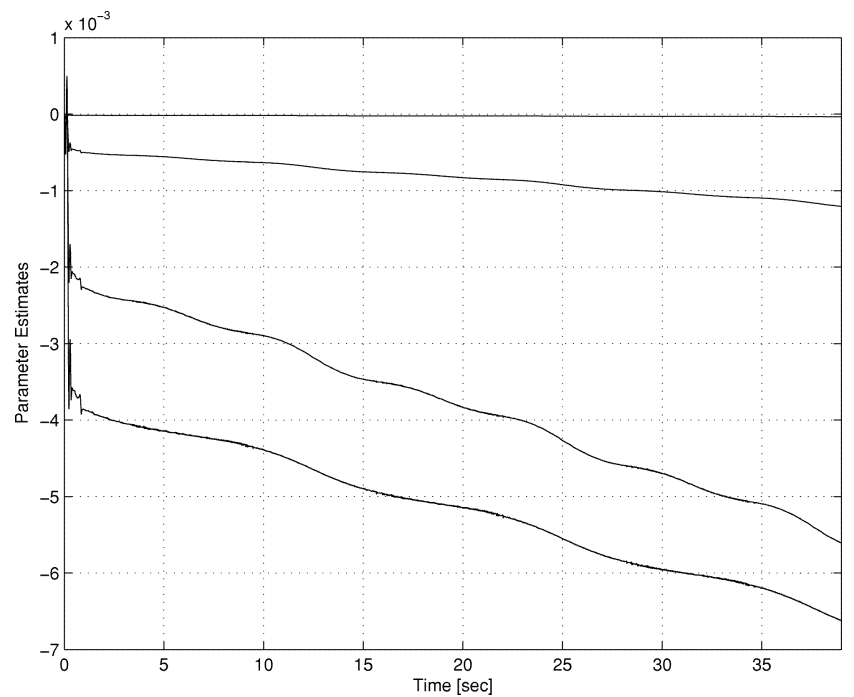

Fig. 7. Parameter estimates for the least-squares update law. 
Remark 4: From Fig. 7, it is clear that some of the parameter estimates to do not converge to constant values. If the lack of estimate convergence becomes problematic for a control designer, a standard projection algorithm (see [1] and [15]) could be applied to bound the estimates to a specified region. A projection algorithm was not utilized for the presented experimental results.

\section{CONCLUSION}

An adaptive torque control input for wheeled mobile robots that can be utilized in a modular manner with parameter estimate update laws has been developed to solve the unified tracking and regulation problems. To achieve this result, we first leveraged off of our previous work in [9] to develop a differentiable kinematic controller that solves the unified tracking and regulation problems and facilitates integrator backstepping. Another motivation for the WMR kinematic control design is that a Lyapunov-based function can be constructed such that its time derivative is negative-definite, and hence, facilitates the modular adaptive control design and stability analysis (e.g., it is not clear how typical WMR kinematic controllers which exploit the use of extended Barbalat's Lemma to prove the stability result (such as [22]), can be utilized in conjunction with the modular adaptive control strategy). After developing the kinematic control design, we then leveraged off of the work of [6] and [13] for robot manipulators, to develop a torque control input that was proven to yield update law modularity. That is, provided a prediction error-based update law ensures the parameter estimate vector is bounded, then all of the signals were proven to be bounded. An additional stability analysis was then provided to prove that if the adaptive update law was designed such that the prediction error is square-integrable and the estimated inertia matrix is positive-definite, the WMR tracking and regulation errors are asymptotically forced to a control term that can be made arbitrarily small. An advantage of the update law modularity is that the control designer is provided with additional flexibility in the design of the adaptive update law. That is, faster parameter convergence, and hence, potentially faster transient performance can be facilitated by various parameter update laws. To demonstrate this advantage, the controller was implemented with prediction error based gradient and least-squares update laws without retuning the controller.

\section{APPENDIX}

\section{PROOF FOR THEOREM 1}

Proof: To prove Theorem 1, we define the nonnegative function $V_{1}(t) \in R$

$$
V_{1}=\frac{1}{2} \eta^{T} M^{*} \eta+\frac{1}{2} w^{2}+\frac{1}{2} \tilde{z}^{T} \tilde{z}
$$

where (55) can be lower and upper bounded as

$$
\lambda_{1}\|\xi\|^{2} \leq V_{1} \leq \lambda_{2} m_{2}\|\xi\|^{2}
$$

where $\lambda_{1}, \lambda_{2} \in R$ are positive bounding constants, $m_{2}(z, w)$ was given in (16), and $\xi(t) \in R^{5}$ is defined as follows:

$$
\xi=\left[\begin{array}{lll}
w & \tilde{z}^{T} & \eta^{T}
\end{array}\right]^{T} .
$$

After taking the time derivative of (55), substituting for the closed-loop error systems given in (38), (42), and (44), utilizing (17), and then cancelling common terms, the following expression is obtained

$$
\begin{aligned}
\dot{V}_{1}= & -k_{a} m_{2} \eta^{T} \eta-k_{1} m_{2} w^{2}-k_{2} m_{2} \tilde{z}^{T} \tilde{z} \\
& +\left[\left\|Y_{s}\right\|_{i \infty}\|\eta\|\|\tilde{\Phi}\|-k_{n 1}\left\|Y_{s}\right\|_{i \infty}^{2}\|\eta\|^{2}\right] \\
& +\left[\left\|\left(\dot{\hat{M}}^{*}-\hat{V}_{m}^{*}\right) \eta\right\|\|\eta\|-k_{n 3}\left\|\left(\dot{\hat{M}}^{*}-\hat{V}_{m}^{*}\right) \eta\right\|^{2}\|\eta\|^{2}\right] \\
& +\left[\left\|\frac{1}{\beta} Y_{f} \dot{\hat{\Phi}}\right\|\|\eta\|-k_{n 2}\left\|\frac{1}{\beta} Y_{f} \dot{\hat{\Phi}}\right\|^{2}\|\eta\|^{2}\right] \\
& +\left[\|J z w\|\|\eta\|-k_{n 4}\|J z w\|^{2}\|\eta\|^{2}\right] \\
& +\left[\|\tilde{z}\|\|\eta\|-k_{n 5}\|\tilde{z}\|^{2}\|\eta\|^{2}\right]
\end{aligned}
$$

where the notation $\|\cdot\|_{i \infty}$ denotes the induced infinity norm of a signal. After completing the squares for the bracketed terms of (58), the following upper bound can be formulated

$$
\begin{aligned}
\dot{V}_{1} \leq-k_{a} m_{2}\|\eta\|^{2}-k_{1} m_{2} w^{2}- & k_{2} m_{2}\|\tilde{z}\|^{2} \\
& +\frac{1}{k_{n 1}}\|\tilde{\Phi}\|^{2}+\sum_{i=2}^{5} \frac{1}{k_{n i}}
\end{aligned}
$$

By utilizing (56), the inequality in (59) can be upper bounded as follows:

$$
\dot{V}_{1} \leq-\gamma_{1} V_{1}+\gamma_{2}
$$

where $\gamma_{1}, \gamma_{2} \in R$ are positive constants defined as

$$
\gamma_{1}=\frac{\min \left(k_{a}, k_{1}, k_{2}\right)}{\lambda_{2}} \quad \gamma_{2}=\frac{1}{k_{n 1}} \sup _{t}\|\tilde{\Phi}(t)\|^{2}+\sum_{i=2}^{5} \frac{1}{k_{n i}}
$$

where the assumption that $\tilde{\Phi}(t) \in L_{\infty}\left[0, t_{f}\right)$ (i.e., a constant bounded value for $\sup _{t}\|\tilde{\Phi}(t)\|^{2}$ exists $\forall t: 0 \leq t<t_{f}$ ) was utilized. After solving the differential inequality given in (60), the following upper bound can be formulated

$$
\begin{array}{r}
V_{1}(t) \leq V_{1}(0) e^{-\gamma_{1} t}+e^{-\gamma_{1} t} \int_{0}^{t} e^{\gamma_{1} \xi} \gamma_{2} d \xi \leq V_{1}(0) e^{-\gamma_{1} t} \\
+\frac{\gamma_{2}}{\gamma_{1}}
\end{array}
$$

Hence, from (55) and (62), the following inequality can be obtained

$$
\|\xi(t)\| \leq \sqrt{\frac{\lambda_{2} m_{2}(z(0), w(0))}{\lambda_{1}}\|\xi(0)\|^{2} e^{-\gamma_{1} t}+\frac{\gamma_{2}}{\gamma_{1} \lambda_{1}}}<\infty .
$$

Based on (57) and (63), it is straightforward to determine that $w(t), \tilde{z}(t), \eta(t) \in L_{\infty}\left[0, t_{f}\right)$. After utilizing (24), (33), and the fact that $\tilde{z}(t), \delta_{d}(t) \in L_{\infty}\left[0, t_{f}\right)$, we can also conclude that $z(t), z_{d}(t) \in L_{\infty}\left[0, t_{f}\right)$. From (8), (10), (25)-(29), (38), and (42), we can prove that $f(t), u_{d}(t), u_{a}(t), \dot{z}_{d}(t), \dot{\tilde{z}}(t), \dot{z}(t), \dot{w}(t)$, $\dot{\eta}(t), \Omega_{1}(t), u(t) \in L_{\infty}\left[0, t_{f}\right)$. Given that $w(t), z(t), u(t) \in$ $L_{\infty}\left[0, t_{f}\right)$, we can utilize (1)-(6), (12), and (13) to prove that $q(t), \dot{q}(t), v(t), \tilde{x}(t), \tilde{y}(t), \tilde{\theta}(t), T(q), \Pi\left(q, q_{r}, v_{r}\right) \quad \in$ 
$L_{\infty}\left[0, t_{f}\right)$. Based on the previous bounding arguments, we can prove that $\dot{u}_{d}(t) \in L_{\infty}$, and hence, $Y_{s}\left(u, u_{d}, \dot{u}_{d}\right), Y_{f}(u) \in$ $L_{\infty}\left[0, t_{f}\right)$. Since $\dot{\eta}(t), \dot{u}_{d}(t) \in L_{\infty}\left[0, t_{f}\right)$, we can utilize the time derivative of (25) to prove that $\dot{u}(t) \in L_{\infty}\left[0, t_{f}\right)$; hence, $Y(u, \dot{u}) \in L_{\infty}\left[0, t_{f}\right)$. Based on the assumption that $\tilde{\Phi}(t) \in L_{\infty}\left[0, t_{f}\right)$, we can now utilize (20) and (21) to prove that $\hat{\Phi}(t), \varepsilon(t), \tau_{f}(t) \in L_{\infty}\left[0, t_{f}\right)$. As described in [7], given that $u(t), \varepsilon(t) \in L_{\infty}\left[0, t_{f}\right)$, we can now prove that $\dot{\hat{\Phi}}(u, \varepsilon) \in L_{\infty}\left[0, t_{f}\right)$; hence, we can now utilize (34) and (35) to prove that $\tau(t), \tau^{*}(t) \in L_{\infty}\left[0, t_{f}\right)$.

\section{APPENDIX \\ PROOF FOR THEOREM 2}

Proof: Theorem 1 can be directly applied to prove that all signals are bounded on $\left[0, t_{f}\right)$ during closed-loop operation. As in [13], the bounds are dependent only on the initial conditions, control gains, and the reference trajectory, (i.e., not dependent on $t_{f}$ ); hence, due to the independence of time, $t_{f}$ can be expanded to $\infty$. To prove Theorem 2, the expressions in (18) and (19) can be used to obtain the following expression

$$
Y \tilde{\Phi}=Y_{s} \tilde{\Phi}-\tilde{M}^{*} \dot{\eta}-\tilde{V}_{m}^{*} \eta
$$

where $\tilde{M}^{*}(q), \tilde{V}_{m}^{*}(q, \dot{q}) \in R^{2 \times 2}$ are defined as follows:

$$
\tilde{M}^{*}=M^{*}-\hat{M}^{*}, \quad \tilde{V}_{m}^{*}=V_{m}^{*}-\hat{V}_{m}^{*}
$$

and $Y_{s}\left(u, u_{d}, \dot{u}_{d}\right) \tilde{\Phi}(t)$ is defined in (19). With the intent of writing the term $Y_{s}\left(u, u_{d}, \dot{u}_{d}\right) \tilde{\Phi}(t)$ in terms of $\varepsilon(t)$, we utilize (20)-(22) to obtain the following expression

$$
\frac{1}{\beta} \dot{\varepsilon}+\varepsilon=\frac{1}{\beta} \dot{Y}_{f} \tilde{\Phi}+Y_{f} \tilde{\Phi}-\frac{1}{\beta} Y_{f} \dot{\hat{\Phi}}=Y \tilde{\Phi}-\frac{1}{\beta} Y_{f} \dot{\hat{\Phi}} .
$$

After substituting (64) into (66) for $Y(u, \dot{u}) \tilde{\Phi}(t)$, the following expression can be obtained

$$
Y_{s} \tilde{\vartheta}=\frac{1}{\beta} \dot{\varepsilon}+\varepsilon+\tilde{M}^{*} \dot{\eta}+\tilde{V}_{m}^{*} \eta+\frac{1}{\beta} Y_{f} \dot{\hat{\Phi}} .
$$

By substituting (67) into (44) and utilizing (65), the closed-loop system given in (44) can be determined as follows:

$$
\hat{M}^{*} \dot{\eta}=-A \eta+\frac{1}{\beta} \dot{\varepsilon}+\varepsilon-\dot{\hat{M}^{*} \eta}
$$

where $A(t) \in R$ is a positive, time-varying signal defined as follows:

$$
\begin{aligned}
A=k_{a} m_{2}+k_{n} & \left(\left\|Y_{s}\right\|_{i \infty}^{2}+\|J z w\|^{2}+\|\tilde{z}\|^{2}\right) \\
& +k_{n}\left\|\frac{1}{\beta} Y_{f} \dot{\hat{\Phi}}\right\|^{2}+k_{n}\left\|\left(\dot{\hat{M}}^{*}-\hat{V}_{m}^{*}\right) \eta\right\|^{2} .
\end{aligned}
$$

To facilitate the subsequent analysis, we define the variable transformation signal $\chi(t) \in R^{2}[6]$ as

$$
\chi=\hat{M}^{*} \eta-\frac{1}{\beta} \varepsilon .
$$

The time derivative of (70) can be determined as

$$
\dot{\chi}=-A \eta+\varepsilon
$$

where (68) was utilized. By utilizing (70), (71) can be rewritten as

$$
\dot{\chi}=-A\left(\hat{M}^{*}\right)^{-1} \chi+\left(I_{n}-\frac{1}{\beta} A\left(\hat{M}^{*}\right)^{-1}\right) \varepsilon .
$$

To examine the stability of $\chi(t)$, another transformation, denoted by $\psi(t) \in R$, is defined as [6]

$$
\psi=\sqrt{\frac{1}{2} \chi^{T} \chi}
$$

After squaring (73) and then taking the time derivative of the resulting expression, the following inequality can be obtained

$$
\begin{aligned}
\frac{d}{d t}\left(\psi^{2}\right)=2 \psi \dot{\psi} \leq & -2 A \lambda_{\min }\left\{\left(\hat{M}^{*}\right)^{-1}\right\} \psi^{2} \\
& +\sqrt{2}\left\|\left(I_{n}-\frac{1}{\beta} A\left(\hat{M}^{*}\right)^{-1}\right)\right\|_{i \infty}\|\varepsilon\| \psi
\end{aligned}
$$

where (72) and (73) were utilized, and $\lambda_{\min }\{\cdot\}$ denotes the minimum eigenvalue of the argument. Based on the definition given in (73), we have that $\psi(t) \geq 0$; hence, (74) can be used to develop the following inequality

$$
\dot{\psi} \leq-\zeta_{1} \psi+\zeta_{2}\|\varepsilon\|
$$

where the nonzero, positive constants $\zeta_{1}, \zeta_{2} \in R$ are defined as follows: ${ }^{2}$

$$
\begin{aligned}
& \zeta_{1}=\inf _{t}\{A\} \lambda_{\min }\left\{\left(\hat{M}^{*}\right)^{-1}\right\} \\
& \zeta_{2}=\frac{\sqrt{2}}{2} \sup _{t}\left\|\left(I_{n}-\frac{1}{\beta} A\left(\hat{M}^{*}\right)^{-1}\right)\right\|_{i \infty} .
\end{aligned}
$$

The solution of the differential inequality of (75) is given by

$$
\psi(t) \leq \psi(0) e^{-\zeta_{1} t}+\zeta_{2} \int_{0}^{t} e^{-\zeta_{1}(t-\xi)}\|\varepsilon(\xi)\| d \xi .
$$

After utilizing Holder's inequality [27], (77) can be rewritten as

$$
\begin{aligned}
|\psi(t)| \leq & |\psi(0)| e^{-\zeta_{1} t}+\zeta_{2}\left(\sqrt{\int_{0}^{t} e^{-\zeta_{1}(t-\xi)} d \xi}\right. \\
& \left.\cdot \sqrt{\int_{0}^{t} e^{-\zeta_{1}(t-\xi)}\|\varepsilon(\xi)\|^{2} d \xi}\right) \\
\leq & |\psi(0)| e^{-\zeta_{1} t}+\frac{\zeta_{2}}{\sqrt{\zeta_{1}}} \sqrt{\int_{0}^{t} e^{-\zeta_{1}(t-\xi)}\|\varepsilon(\xi)\|^{2} d \xi} .
\end{aligned}
$$

After squaring (78) and integrating the resulting expression, the following expression can be obtained

$$
\begin{aligned}
\int_{0}^{t}|\psi(\sigma)|^{2} d \sigma & \leq \frac{|\psi(0)|^{2}}{\zeta_{1}} \\
& +\frac{2 \zeta_{2}^{2}}{\zeta_{1}} \int_{0}^{t}\left(\int_{0}^{\sigma} e^{-\zeta_{1}(\sigma-\xi)}\|\varepsilon(\xi)\|^{2} d \xi\right) d \sigma
\end{aligned}
$$

${ }^{2}$ Provided $\hat{M}(q)$ is positive definite, $\hat{M}^{-1}(q)$ will also be positive definite, and hence, the minimum eigenvalue will be positive. Moreover, since all signals are bounded from the previous analysis, the time-varying signals $A(t)$ and $\hat{M}^{-1}(q(t))$ can be bounded by constants. 
where the fact that

$$
\begin{aligned}
& \left(|\psi(0)| e^{-\zeta_{1} t}+\frac{\zeta_{2}}{\sqrt{\zeta_{1}}} \sqrt{\int_{0}^{t} e^{-\zeta_{1}(t-\xi)}\|\varepsilon(\xi)\|^{2} d \xi}\right)^{2} \\
& \leq 2\left(\left|\psi^{2}(0)\right| e^{-2 \zeta_{1} t}\right. \\
& \left.\quad+\frac{\zeta_{2}^{2}}{\zeta_{1}} \int_{0}^{t} e^{-\zeta_{1}(t-\xi)}\|\varepsilon(\xi)\|^{2} d \xi\right)
\end{aligned}
$$

has been utilized. After reversing the order of integration in (79), the following expression is obtained

$$
\begin{aligned}
\int_{0}^{t}|\psi(\sigma)|^{2} d \sigma \leq & \frac{|\psi(0)|^{2}}{\zeta_{1}}+\frac{2 \zeta_{2}^{2}}{\zeta_{1}} \int_{0}^{t} e^{\zeta_{1} \xi}\|\varepsilon(\xi)\|^{2} \\
& \cdot\left(\int_{\xi}^{t} e^{-\zeta_{1} \sigma} d \sigma\right) d \xi \\
\leq & \frac{|\psi(0)|^{2}}{\zeta_{1}}+\frac{2 \zeta_{2}^{2}}{\zeta_{1}} \int_{0}^{t}\|\varepsilon(\xi)\|^{2} \frac{1}{\zeta_{1}} d \xi
\end{aligned}
$$

Based on the assumption that $\dot{\hat{\Phi}}(u, \varepsilon)$ is designed to ensure that $\varepsilon(t) \in L_{2}$, we can utilize (81) to prove that

$$
\|\psi\|_{2} \leq \frac{|\psi(0)|^{2}}{\zeta_{1}}+\frac{2 \zeta_{2}^{2}}{\zeta_{1}^{2}}\|\varepsilon\|_{2}<\infty
$$

where $\|\cdot\|_{2}$ denotes the $L_{2}$ norm of a signal. From (82), we can conclude that $\psi(t) \in L_{2}$; hence, from (73), we can prove that $\chi(t) \in L_{2}$. Based on the results from Theorem 1, we can utilize (69) and (71) to prove that $A(t), \dot{\chi}(t) \in L_{\infty}$. After taking the time derivative of (70) and utilizing the fact that $\dot{\eta}(t), \dot{\hat{M}}^{*}(u, \varepsilon), \dot{\chi}(t) \in L_{\infty}$, we can conclude that $\dot{\varepsilon}(t) \in L_{\infty}$. Since $\chi(t), \varepsilon(t) \in L_{\infty} \cap L_{2}$ and $\dot{\chi}(t), \dot{\varepsilon}(t) \in L_{\infty}$, we can use Barbalat's Lemma [25] to prove that

$$
\lim _{t \rightarrow \infty} \chi(t), \varepsilon(t)=0
$$

and hence, from (70), we can prove that

$$
\lim _{t \rightarrow \infty} \eta(t)=0 \text {. }
$$

From (55), (58), and (84), we can now determine that

$$
\lim _{t \rightarrow \infty} \tilde{z}(t), w(t)=0 \text {. }
$$

Based on the result given in (85), the triangle inequality can be applied to (24) to prove that

$$
\lim _{t \rightarrow \infty}\|z\|=\delta_{d}(t)
$$

where (33) was utilized. By utilizing (30), (85), and (86), the result given in (45) be be obtained from taking the inverse of the transformation given in (6).

\section{REFERENCES}

[1] M. M. Bridges, D. M. Dawson, and C. T. Abdallah, "Control of rigid-link flexible-joint robots: A survey of backstepping approaches," J. Robot. Sys., vol. 12, no. 3, pp. 199-216, 1995.

[2] R. Brockett, "Asymptotic stability and feedback stabilization," in Differential Geometric Control Theory, R. Brockett, R. Millman, and H. Sussmann, Eds. Boston, MA: Birkhauser, 1983.
[3] C. Canudas de Wit and O. Sordalen, "Exponential stabilization of mobile robots with nonholonomic constraints," IEEE Trans. Automat. Contr., vol. 37, pp. 1791-1797, Nov. 1992.

[4] J. Coron and J. Pomet, "A remark on the design of time-varying stabilizing feedback laws for controllable systems without drift," in Proc. IFAC Symp. Nonlinear Contr. Syst. Design (NOLCOS), 1992, pp. 413-417.

[5] J. J. Craig, P. Hsu, and S. Sastry, "Adaptive control of mechanical manipulators," in IEEE Conf. Robot. Automat., 1986, pp. 190-195.

[6] M. S. de Queiroz, D. M. Dawson, and M. Agarwal, "Adaptive control of robot manipulators with controller/update law modularity," Automatica, vol. 35, no. 8, pp. 1379-1390, 1999.

[7] M. S. de Queiroz, D. M. Dawson, S. Nagarkatti, and F. Zhang, LyapunovBased Control of Mechanical Systems. Boston, MA: Birkhäuser, 2000.

[8] W. E. Dixon, D. M. Dawson, E. Zergeroglu, and A. Behal, Nonlinear Control of Wheeled Mobile Robots, Vol. 262, Lecture Notes in Control and Information Sciences. New York: Springer-Verlag, 2000.

[9] W. E. Dixon, D. M. Dawson, E. Zergeroglu, and F. Zhang, "Robust tracking and regulation control for mobile robots," Int. J. Robust and Nonlinear Contr., vol. 10, pp. 199-216, 2000.

[10] W. E. Dixon, D. M. Dawson, F. Zhang, and E. Zergeroglu, "Global exponential tracking control of a mobile robot system via a PE condition," IEEE Trans. Syste. Man, and Cyber-Part B: Cyber., vol. 30, pp. $129-142,2000$

[11] W. Dong and W. Huo, "Adaptive stabilization of dynamic nonholonomic chained systems with uncertainty," in Proc. 36th IEEE Conf. Decision and Contr., 1997, pp. 2362-2367.

[12] P. Kokotovic, "The joy of feedback: Nonlinear and adaptive," IEEE Contr. Syst. Mag., vol. 12, pp. 7-17, 1992.

[13] M. Krstic and P. V. Kokotovic, "Adaptive nonlinear design with controller-identifier separation and swapping," IEEE Trans. Automat. Contr., vol. 40, pp. 426-440, 1995.

[14] R. L. Leal and C. Canudas de Wit, "Passivity based adaptive control for mechanical manipulators using LS-type estimation," IEEE Trans. Automat. Contr., vol. 35, pp. 1363-1365, 1990.

[15] R. L. Leal and B. Brogliato, "Adaptive control of robot manipulators with flexible joints," IEEE Trans. Automat. Contr., vol. 37, pp. 174-181, 1992.

[16] F. L. Lewis, C. T. Abdallah, and D. M. Dawson, Control of Robot Manipulators. New York: MacMillan, 1993.

[17] M. S. Loffler, N. P. Costescu, and D. M. Dawson, "QMotor 3.0 and the QMotor robotic toolkit: A PC-based control platform," IEEE Contr. Syst. Mag., vol. 22, no. 3, June 2002.

[18] R. McCloskey and R. Murray, "Exponential stabilization of driftless nonlinear control systems using homogeneous feedback," IEEE Trans. Automat. Contr., vol. 42, pp. 614-628, May 1997.

[19] R. H. Middleton and C. G. Goodwin, "Adaptive computed torque control for rigid link manipulators," Syst. Contr. Lett., vol. 10, pp. 9-16, 1988.

[20] J. Pomet, "Explicit design of time-varying stabilizing control laws for a class of controllable systems without drift," Syst. Contr. Lett., vol. 18, no. 2, pp. 147-158, 1992.

[21] N. Sadegh and R. Horowitz, "An exponentially stable adaptive control law for robot manipulators," IEEE Trans. Robot. Automat., vol. 6, pp. 491-496, 1990

[22] C. Samson, "Velocity and torque feedback control of a nonholonomic cart," presented at the Proc. Int. Workshop in Adaptive and Nonlinear Control: Issues in Robotics, Grenoble, France, 1990.

[23] — " "Control of chained systems application to path following and time-varying point-stabilization of mobile robots," IEEE Trans. Automat. Contr., vol. 40, pp. 64-77, 1997.

[24] A. Teel, R. Murray, and C. Walsh, "Nonholonomic control systems: From steering to stabilization with sinusoids," Int. J. Contr., vol. 62, no. 4, pp. 849-870, 1995.

[25] J. J. E. Slotine and W. Li, Applied Nonlinear Control. Englewood Cliffs, NJ: Prentice-Hall, 1991.

[26] Y. Tang and M. A. Arteaga, "Adaptive control of robot manipulators based on passivity," IEEE Trans. Automat. Contr., vol. 39, pp. 1871-1875, 1994.

[27] M. Vidyasagar, Nonlinear Systems Analysis. Englewood Cliffs, NJ: Prentice-Hall, 1978.

[28] E. Zergeroglu, W. E. Dixon, D. Haste, and D. M. Dawson, "Composite adaptive output feedback tracking control of robotic manipulators," Robotica, vol. 17, pp. 591-600, 1999. 